\title{
Bilateral Yokoyama procedure in a patient with esotropic strabismus fixus due to severe myopia
}

\author{
Katarzyna Pelińska', Valeriia Peredysta ${ }^{1}$, Magdalena Sildatke-Bauer ${ }^{2}$, Piotr Loba ${ }^{1}$ \\ 'Department of Binocular Vision Pathophysiology and Strabismus, First Chair of Ophthalmology, Medical University of Lodz, Poland \\ ${ }^{2}$ Strabismus Outpatient Clinic, St. John Hospital, Starogard Gdański, Poland
}

\begin{abstract}
The aim of this case report is to present successful management of bilateral esotropic strabismus fixus due to severe myopia in a 63-year-old female patient. Myopic strabismus fixus is a rare condition that develops in patients with high myopia due to enlarged dimensions of the globe. This type of ocular misalignment may progress from small angle esotropia with free ocular movements, to the stage in which one or both eyes are anchored in a position of extreme adduction. In the case presented, esotropia
\end{abstract}

was so severe that both eyes were fixed in an adducted position. The patient had to adopt a severe head turn in order to make use of her better eye; nevertheless her visual function was poor. We performed a bilateral Yokoyama procedure with simultaneous medial rectus recession. The surgery resulted in a large improvement of ductions and reduction of esotropia. The patient's visual function improved substantially and the head turn was vastly reduced. KEY WORDS: myopic strabismus fixus, high myopia, large angle esotropia, Yokoyama's surgery.

\section{INTRODUCTION}

Myopic strabismus fixus (MSF) is a consequence of very high myopia, also called heavy eye syndrome (HES), which is characterized by esotropia and hypotropia [1]. It usually occurs in elderly patients and is progressive in nature. The severity of strabismus is related to the axial length of the eye. A great number of patients tend to have myopia larger than $15 \mathrm{D}$ and the axial length longer than $30 \mathrm{~mm}$ [2]. The growing eye has very weak orbital septa especially between the superior rectus muscle (SR) and medial rectus muscle and between the lateral rectus muscle (LR) and inferior rectus muscle. This is the reason why the lateral rectus is displaced inferiorly and the superior rectus translocates nasally [1].

The disease is usually bilateral but can be asymmetric. Limitation of abduction of the eye induces diplopia and head turn. Subsequently patients start to use their better eye. It is rather rare to see a patient with such severe duction limitation that their visual functioning is impaired. Usually the problem is managed at an early or intermediate stage.

A number of procedures have been proposed for HES including hemitransposition of rectus muscle [3], partial Jensen's operation [4] and loop myopexy [5, 6], initially described by Yokoyama. The effectiveness of this procedure is related to the fact that it addresses the true cause of the prob- lem, which is the displacement of superior and lateral rectus muscles [7].

\section{CASE PRESENTATION}

A 63-year-old female patient was referred due to severe esotropia with hypotropia of both eyes. Ductions were severely limited with the right eye being in a fixed adducted position (Figure 1). She could practically use her eyes only if she adopted a large left head turn but could not keep it that way for long. She did not report diplopia. She could not move around without the help of other people, which in consequence made her functionally blind.

The pathological myopia appeared in early childhood and was not properly corrected with glasses. At the age of 47 years the patient underwent bilateral refractive lens extraction. The best corrected visual acuity improved then from hand movement to 0.1 with -6.0 Dsph -5.0 Dcyl ax 6 correction in the right eye and to 0.04 with -2.25 Dsph -5.0 Dcyl ax 99 correction in the left eye. In the past 3 years the patient's right eye was in extreme adduction with no movements. The left eye was adducted as well but preserved some, very limited movements.

At admission visual acuity of both eyes was 0.05 BCVA. It was impossible to perform automatic refraction. Orthoptic

\section{CORRESPONDING AUTHOR}

Katarzyna Pelińska, Department of Binocular Vision Pathophysiology, First Chair of Ophthalmology, Medical University of Lodz, 22 Kopcińskiego St., $90-153$ Lodz, Poland, e-mail: katarzynapelinska@gmail.com 


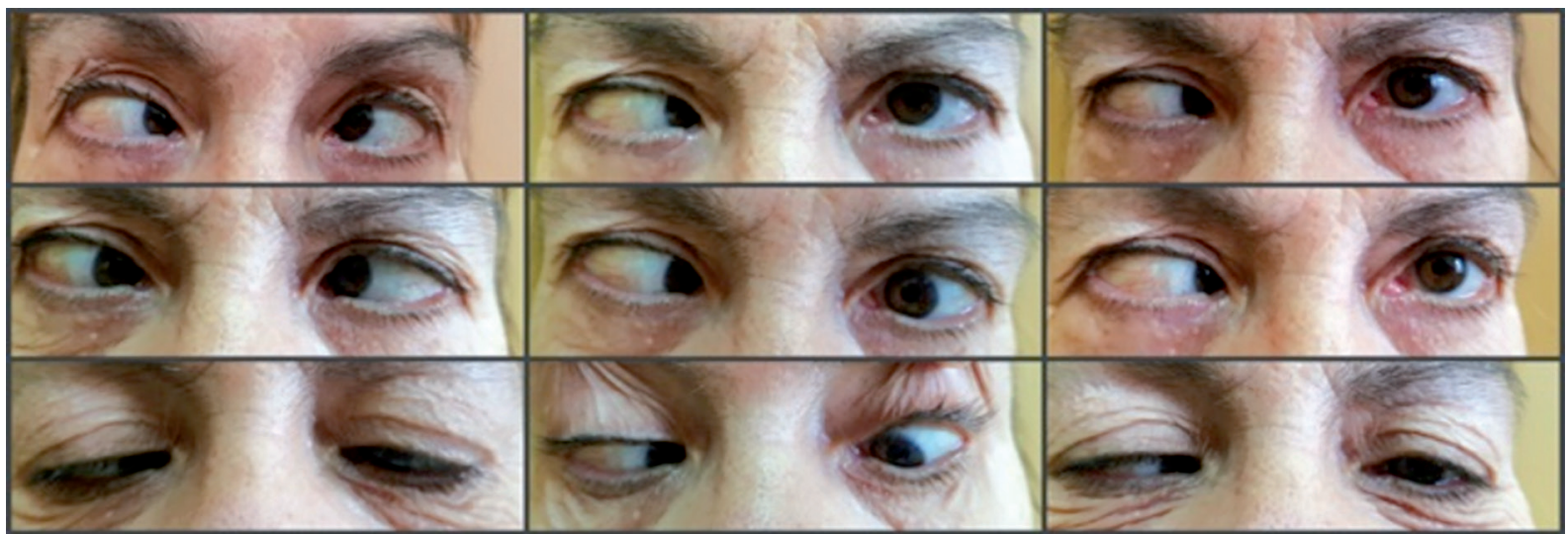

Figure 1. Preoperative photograph shows limitation of abduction and supraduction of both eyes, especially right. In primary position patient showed more than 100 prism diopters

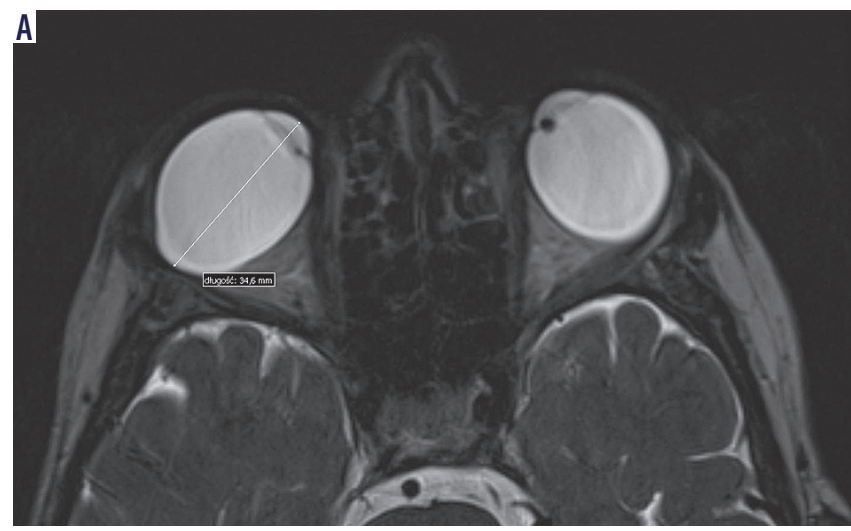

Figure 2. MRI scan. A) Axial length of the globes. Right eye $-34.6 \mathrm{~mm}$, left eye $-31.9 \mathrm{~mm}$. B) Coronal scan shows displacement of lateral rectus downward and superior rectus medially

examination showed limitation of extraocular movements of both eyes and over 100 prism diopters (PD) esotropia with right hypotropia.

Slit lamp examination showed normal anterior segment and postoperative pseudophakia. Examination of the posterior segment revealed retinal pigment epithelial thinning and diffuse chorioretinal atrophy. Tilted disc with peripapillary atrophy was observed.

Orbital MRI scan of the extraocular muscles was performed. The elongated oval eyeballs were found with superotemporal globe prolapse which displaced the lateral rectus inferiorly and superior rectus nasally; both muscles were significantly thickened. Axial length of the right eye was $34.6 \mathrm{~mm}$ and of the left one $31.9 \mathrm{~mm}$. In both eyes an atypical slanting position of the optic nerves was observed (Figure 2A, B).

Due to the above findings the decision to perform bilateral Yokoyama's surgery was undertaken with the patient's consent. The surgery was performed in general anesthesia. First the forced duction test (FDT) was performed, revealing

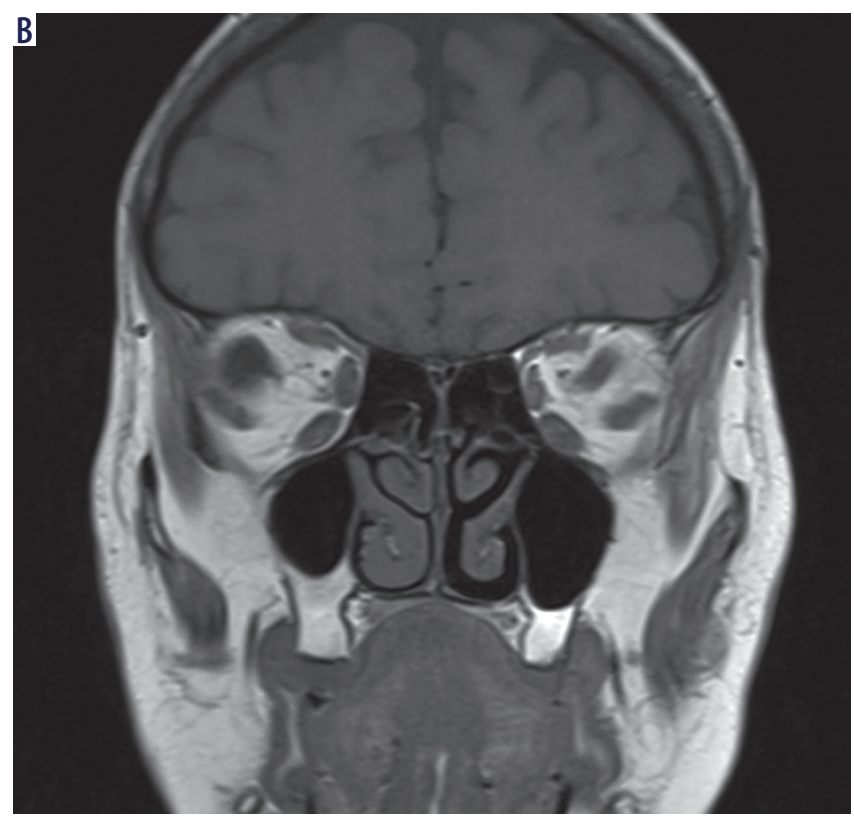

restriction of abduction in both eyes. The conjunctiva was opened near the limbus at a 270-degree angle. The superior rectus muscle and lateral rectus muscle were isolated with the muscle hooks. The distance between the muscle bellies was abnormally high (Figure 3).

Around $14 \mathrm{~mm}$ posteriorly from the muscle insertion a non-absorbable Polyester 5.0 suture was passed through the upper half of the lateral rectus muscle and the temporal half of the superior rectus muscle bellies and knotted together (Figure 4). The same technique was performed on the left eye. Subsequently the bilateral recession of medial rectus muscle was performed (in right eye $8 \mathrm{~mm}$, in left eye $6 \mathrm{~mm}$ ) with non-adjustable Vicryl 6.0 sutures. The conjunctiva was closed with 8.0 Vicryl single sutures.

The day after surgery the angle of esodeviation measured with the prism cover test decreased to $60 \mathrm{PD}$ with slight right hypotropia. During 2 weeks of observation postoperative results were stable (Figure 5). Improvement in elevation and abduction was observed. Visual acuity also improved to $0.16 \mathrm{cc}$ -9.0 Dsph in the right and $0.08 \mathrm{cc}-2.5 \mathrm{Dsph}-4.0 \mathrm{Dcyl}$ ax 108 in 


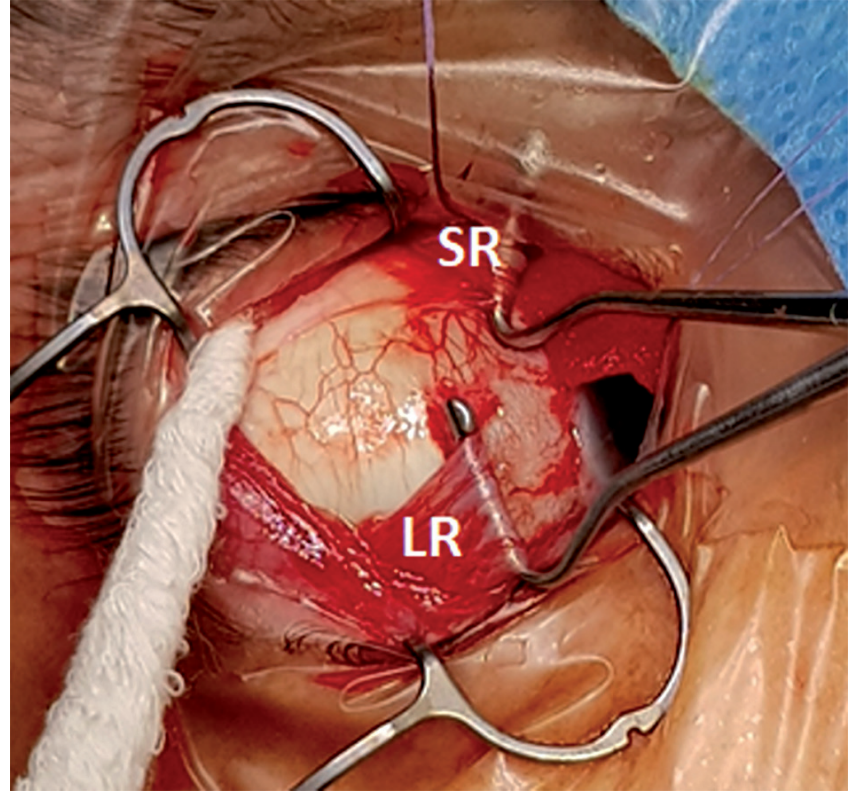

Figure 3. Intraoperative findings. The superior rectus muscle (SR) and lateral rectus muscle (LR) were isolated with the muscle hooks

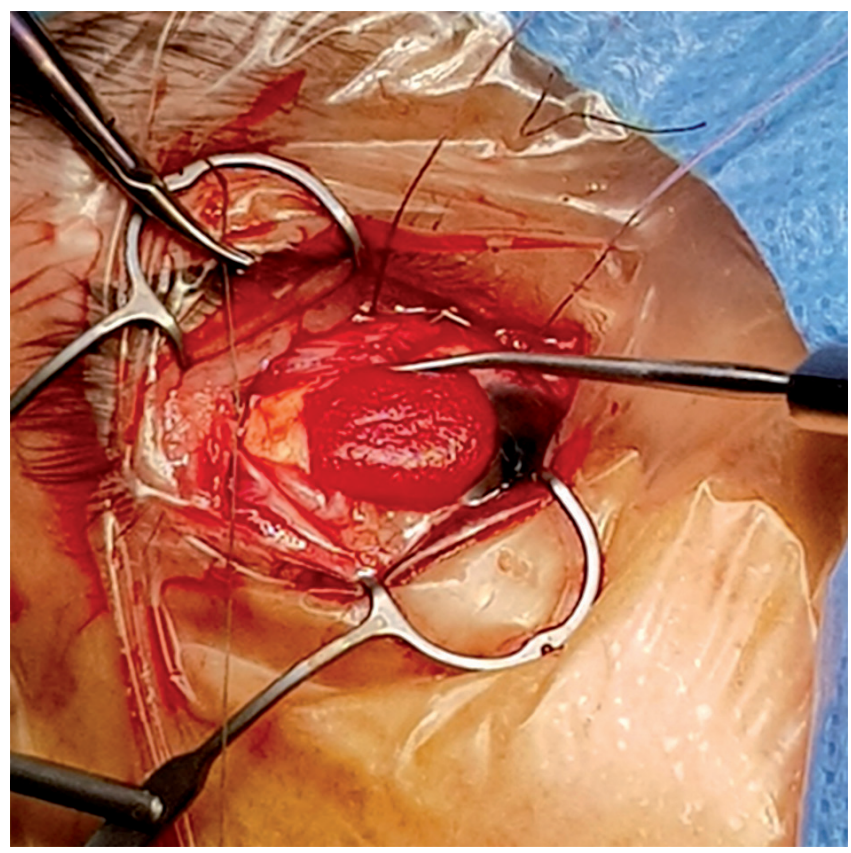

Figure 4. Intraoperative findings. Creation of surgical connection between the superior rectus and lateral rectus muscle with 5.0 Polyester suture the left eye. The patient noticed a subjective improvement in general vision as early as the day after the operation. In two months observation the angle of deviation remained stable and was $60 \mathrm{PD}$. Visual acuity improved, reaching in the right eye $0.2 \mathrm{cc}-7.00-2.5$ ax 50 and the left eye $0.1 \mathrm{cc}-2.00-5.0$ ax 100. The patient reported a significant positive change in her quality of life and mood, especially indicating an increased feeling of independence - nowadays she does not require help from other people in daily activities. Her anxiety and depression have decreased. We reported a large positive change in her self-esteem as well. According to the patient's words she experienced a great benefit from the surgery.

As further improvement was not considered possible, the patient has remained under follow-up.

\section{DISCUSSION}

Heavy eye syndrome, also called myopic strabismus fixus, is a very rare type of restrictive strabismus which develops in highly myopic individuals. The term "heavy eye" was used to describe the sensation that a bigger, heavier myopic eye sinks in orbital tissues and its anterior part is decreased.

For the first time, the clinical features of this condition were described by Huggonnier and Magnard in 1969. They suggested a correspondence with structural lesions, such as fibrosis of the lateral rectus muscle [8]. Another hypothesis was proposed by Bagolini, who suggested that the reason for the LR myopathy lies in the compression of the muscle against the lateral orbital wall by the enlarged myopic globe [9]. He noted that the highly myopic eye globe enlarges in adulthood long after the orbital bone growth cessation, resulting in a potential increased pressure on the LR.

Later computed tomography (CT) started to play an essential role in assessing HES. Among the first to use CT to support their hypothesis were Demer and Von Noorden. CT scans allowed for quantitative assessment of the orbital anatomy and revealed mechanical restriction between the posterior part of the globe and bones of the orbital apex, which could be the possible cause of strabismus in HES [10]. Kowal et al. were the first to publish MRI scans of HES [11]. Krzizok and Schroeder published a paper on this subject implying that there are displacements in all the rectus muscles' paths, with particular inferior displacement of the lateral rectus muscle [7].

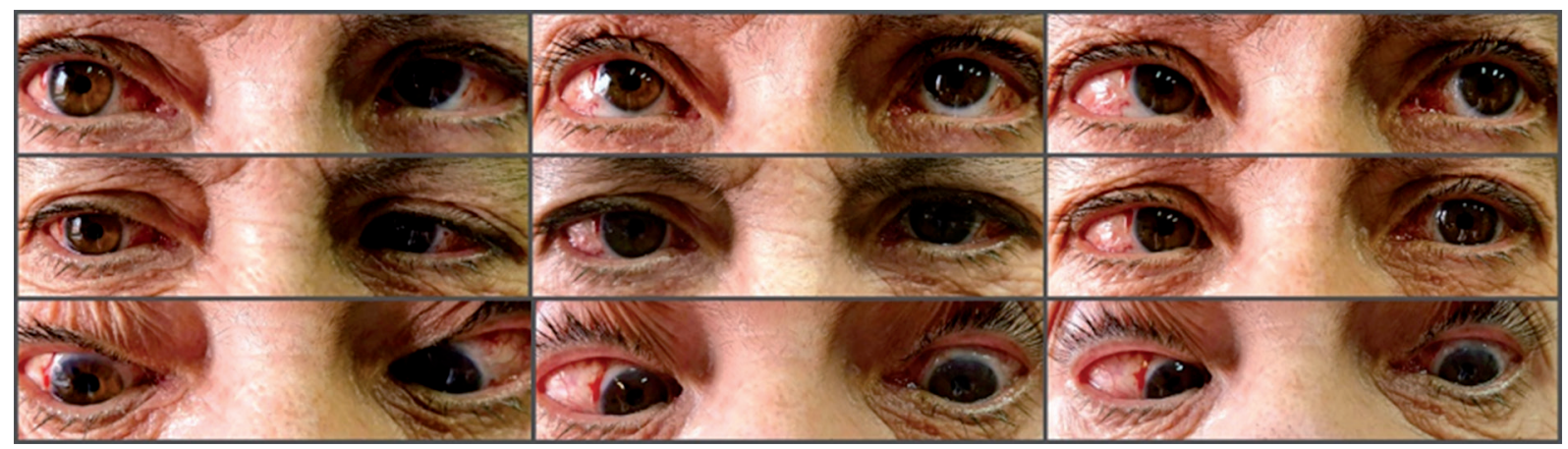

Figure 5. Two-weeks after Yokoyama procedure on both of the eyes. Photograph demonstrates reduced esotropia in primary position and improvement in all gaze positions 
Yokoyama provided the most recent explanation, stating that the enlarged globe in high degree myopia herniates superotemporally and retroequatorially through the muscle cone because of the weak septa. It means that the pathogenesis of this condition lies not only in downward displacement of the lateral rectus muscle, as some authors claimed before, but also in dislocation of the elongated eyeball out of the muscle cone [12]. These findings were also confirmed by Aoki et al. [13]. Nowadays this is the most accepted theory, confirmed not only in MRI imaging but also by surgical feedback.

A very similar condition, but in elderly patients usually nonmyopic, is known as sagging eye syndrome (SES), first described by Rutar and Demer [14]. In SES distance esotropia and cyclovertical strabismus with limited supraduction is observed, as a result of age-related involution of orbital connective tissue [15]. Degeneration of the LR-SR band leads only to inferior LR displacement (SR is not displaced). Unlike in HES, no superotemporal globe prolapse, which is an anatomical hallmark for HES, is seen. That is why preoperative orbital imaging has to be performed to confirm the diagnosis. It is essential to distinguish this age-related strabismus from HES, because the way of treatment is different. Even in highly myopic patients with SES conventional surgery, an augmented medial rectus muscle recession is an appropriate choice, whereas HES requires formation of a surgical union between the SR and LR muscles.

Yokoyama's surgery offers treatment for progressive esotropia in highly myopic patients by suturing superior rectus and lateral rectus bellies. If large-angle esotropia with severely limited eye movements is present in both eyes, then Yokoyama's procedure should be performed bilaterally. Due to the fact that only half of each muscle is mobilized and transposed, the risk of anterior ischemia is relatively low [16]. Although for treating esotropia the medial rectus recession is a standard procedure, it may not be necessary in progressive esotropia caused by pathological myopia. The ipsilateral recession of medial rectus muscle should be performed only if FDT still shows restriction of abduction despite Yokoyama's procedure. Resection of the lateral rectus muscle, which is also a routine procedure for ordinary esotropia, should be avoided in this case.

\section{CONCLUSIONS}

Patients with an acquired strabismus in highly myopic eyes should always undergo orbital imaging. If a downward lateral rectus displacement and nasal superior rectus displacement with severe superotemporal globe prolapse are observed, the diagnosis of HES should be considered. Clinical features of HES are progressive esotropia and hypotropia with mechanically restricted eye movements, especially abduction and supraduction.

Over the years, many operative methods to stabilize the globe in high myopia have been suggested. Traditionally performed procedures of resection - recession or the traction suture - may be effective in some cases, but there is big risk of recurrence unlike in Yokoyama's surgery. This case study proves that Yokoyama's surgery is a very effective type of surgical intervention as significant improvement in psychosocial and functional quality of the patient's life was observed.

\section{DISCLOSURE}

The authors declare no conflict of interest.

\section{References}

1. Yamaguchi M, Yokoyama T, Shiraki K. Surgical procedure for correcting globe dislocation in highly myopic strabismus. Am J Ophthalmol 2010; 149: 341-346.

2. Krzizok TH, Kaufmann H, Traupe H. New approach in strabismus surgery in high myopia. Br J Ophthalmol 1997; 81: 625-630.

3. Yamada M, Taniguchi S, Muroi T, et al. Rectus eye muscle paths after surgical correction of convergent strabismus fixus. Am J Ophthalmol 2002; 134: 630-632.

4. Larsen PC GGA, Larsen PC, Gole GA. Partial Jensen's procedure for the treatment of myopic strabismus fixus. J AAPOS 2004; 8: 393-395.

5. Durnian JM, Maddula S, Marsh IB. Treatment of "heavy eye syndrome" using simple loop myopexy. J AAPOS 2010; 14: 39-41.

6. Wong I, Leo SW, Khoo BK. Loop myopexy for treatment of myopic strabismus fixus. J AAPOS 2005; 9: 589-591.

7. Krzizok TH, Schroeder BU. Measurement of recti eye muscle paths by magnetic resonance imaging in highly myopic and normal subjects. Invest Ophthalmol Vis Sci 1999; 40: 2554-2560.

8. Hugonnier R, Magnard P. Les desequilibres oculo-moteurs observès en cas de myopie forte. Ann Ocul (Paris) 1969; 202: 713-724.

9. Bagolini B, Tamburrelli C, Dickmann A, et al. Convergent strabismus fixus in high myopic patients. Doc Ophthalmol 1990; 74: 309-320.

10. Demer JL, Von Noorden GK. High myopia as an unusual cause of restrictive motility disturbance. Surv Ophthalmol 1989; 33: 281-284.

11. Kowal L, Troski M, Gilford E. MRI in the heavy eye phenomenon. Aust N Z J Ophthalmol 1994; 22: 125-126.

12. Yokoyama T. Ocular motility abnormalities. In: Spaide RF, Ohno-Matsui K, Yannuzzi LA. Pathologic Myopia. Springer New York, New York, NY 2014; 323-332.

13. Aoki Y, Nishida Y, Hayashi 0, et al. Magnetic resonance imaging measurements of extraocular muscle path shift and posterior eyeball prolapse from the muscle cone in acquired esotropia with high myopia. Am J Ophthalmol 2003; 136: 482-489.

14. Rutar T, Demer JL. "Heavy Eye" syndrome in the absence of high myopia: A connective tissue degeneration in elderly strabismic patients. J AAPOS 2009; 13: 36-44.

15. Tan RJD, Demer JL. Heavy eye syndrome versus sagging eye syndrome in high myopia. J AAPOS 2015; 19: 500-506.

16. Pineles SL, Chang MY, Oltra EL, et al. Anterior segment ischemia: etiology, assessment, and management. Eye (Lond) 2018; 32: 173-178. 